\title{
Depressão materna e riscos para o comportamento e a saúde mental das crianças: Uma revisão
}

\author{
Fernanda Aguiar Pizeta \\ Tribunal de Justiça do Estado de São Paulo \\ Universidade de São Paulo - Ribeirão Preto \\ Thaysa Brinck Fernandes Silva \\ Universidade de São Paulo - Ribeirão Preto \\ Maria Isabela Brasil Cartafina \\ Psicóloga \\ Sonia Regina Loureiro \\ Universidade de São Paulo - Ribeirão Preto
}

\begin{abstract}
Resumo
A depressão materna tem sido considerada um fator de risco para a saúde mental das crianças. Objetivouse identificar e analisar na literatura indexada artigos empíricos recentes (2005 a 2012), que abordaram as associações da depressão materna ao comportamento e à saúde mental de crianças, escolares. Procedeu-se a uma busca sistemática nas bases PubMed, PsycInfo e LILACS, por meio das palavras-chave "Maternal Depression or Depression" e "Risk Factors". Foram selecionados e analisados 68 artigos, 23 transversais e 45 longitudinais. Independentemente dos delineamentos adotados, a depressão materna mostrou-se associada à presença de dificuldades emocionais e comportamentais em geral, de manifestações depressivas e de ansiedade. Além da depressão materna, outras variáveis contextuais mostraram-se associadas às dificuldades das crianças. O reconhecimento da ação de múltiplas variáveis permitiu a verificação de indicadores diversos, o que pode favorecer o planejamento de intervenções.
\end{abstract}

Palavras-chave: depressão materna; crianças; comportamento; saúde mental; fatores de risco.

\begin{abstract}
Maternal depression and risks for children's behavior and mental health: A review. Maternal depression has been considered a risk factor for children's mental health. The aim of this review was to identify and analyze in the related literature, recent empirical studies (2005-2012), that address the associations of maternal depression to the children's behavior and mental health, at school age. The search has been held in the databases PubMed, PsycInfo and LILACS, using the keywords "Maternal Depression or Depression" and "Risk Factors". It was identified and analyzed 68 articles, 23 with cross-sectional and 45 with longitudinal design. Regardless of the designs adopted, maternal depression was associated with the presence of emotional and behavioral difficulties in general, and depressive and anxiety manifestations. Besides maternal depression, other contextual variables were related to children's difficulties. The recognition of multiples variables' action allowed the verification of several indicators, which can favor the planning of interventions.
\end{abstract}

Keywords: maternal depression; children; behavior; mental health; risk factors.

\section{Resumen}

La depresión materna y los riesgos para el comportamiento y la salud mental de los niños: Una revisión. La depresión materna se ha considerado un factor de riesgo para la salud mental de los niños. El objetivo fue identificar y analizar en la literatura relacionada los estudios empíricos recientes (2005-2012) que se ha abordado las asociaciones de la depresión materna con el comportamiento y la salud mental de los niños, en la edad escolar. La búsqueda sistemática se ha realizado en las bases de datos PubMed, PsycINFO y LILACS, utilizando las palabras clave "Maternal Depression or Depression" y "Risk Factors". Se seleccionaron y analizaron 68 artículos, 23 transversales y 45 longitudinales. La depresión materna se asoció con la presencia de dificultades emocionales y de comportamiento en general, de manifestaciones de ansiedad y depresión. Además de la depresión materna, otras variables contextuales estaban relacionadas con las dificultades de los niños. El reconocimiento de la acción de múltiples variables permitió la verificación de varios indicadores, que puede favorecer la planificación de las intervenciones.

Palabras clave: depresión materna; niños; comportamiento; salud mental; factores de riesgo. 
$\mathrm{O}$ $\mathrm{s}$ transtornos mentais configuram-se como problemas de grande relevância para a população mundial, dada a sua prevalência, incidência nas diferentes faixas etárias e prejuízos a eles associados relativos ao indivíduo, à família e à comunidade.

Estudos epidemiológicos têm demonstrado que a frequência de ocorrência e o curso dos transtornos mentais estão associados, entre outros fatores, à questão de gênero. Com relação aos sintomas depressivos e ansiosos, as mulheres têm sido consideradas mais vulneráveis que os homens, apresentando maiores taxas de prevalência de transtornos de humor e ansiedade. Dentre os transtornos de humor, a depressão configura-se como o transtorno que mais causa incapacitação em mulheres, tanto em países desenvolvidos como em países em desenvolvimento (Andrade, Viana, \& Silveira, 2006).

Considerando-se a prevalência da depressão em mulheres, a condição recorrente, característica do transtorno e a maior incidência no início da idade adulta, a qual coincide com a idade fértil, muitas mulheres acometidas pela depressão são mães, sendo que tal sintomatologia pode interferir negativamente na função materna e no desenvolvimento de seus filhos (Markowitz, 2008).

Os prejuízos no desenvolvimento das crianças, tais como as dificuldades comportamentais e os problemas de saúde mental, são pouco identificados em todas as partes do mundo, o que torna difícil determinar a sua magnitude (Belfer, 2008). Contudo, reconhece-se que tais problemas, além de favorecerem prejuízos para a adaptação da criança, podem também potencializar o risco de transtornos psicossociais na vida adulta (Ferriolli, Marturano, \& Puntel, 2007).

$\mathrm{Na}$ tentativa de melhorar a identificação precoce dos problemas de saúde mental infantil, diversos estudos têm se ocupado das condições contextuais de risco, tais como a exposição a eventos ambientais adversos (Halpern \& Figueiras, 2004), sendo que a depressão materna pode ser vista como uma dessas condições de adversidade.

Neste estudo, pretendeu-se verificar, com base em uma revisão sistemática da literatura, o estado da arte quanto ao conhecimento sobre como a depressão materna associa-se à saúde mental das crianças. Objetivou-se identificar e analisar estudos empíricos recentes que abordaram as associações da depressão materna ao comportamento e à saúde mental infantil, verificando também as associações com outras condições contextuais de risco, tendo como foco de interesse crianças em idade escolar.

\section{Método}

Procedeu-se, inicialmente, a uma busca exclusivamente de artigos empíricos indexados, identificados nas bases de dados PubMed, LILACS e PsycInfo, no período de 2005 a 2012, por meio das palavras-chave: "Maternal Depression ou Depression" e "Risk Factors", e seus correspondentes em português. Em uma segunda etapa, refinou-se a busca por meio da leitura sistemática dos resumos dos 452 artigos identificados inicialmente, procedendo-se às inclusões e exclusões.

Adotaram-se como critérios de inclusão os idiomas inglês, português e espanhol, população de humanos e como faixa etária de 06 a 12 anos (escolares), como momento de avaliação, incluindo-se artigos em que as crianças foram também avaliadas na idade pré-escolar e/ou nas etapas posteriores do desenvolvimento. Para as exclusões, consideraram-se como principais motivos: estudos com avaliações exclusivas de aspectos clínicos da depressão em crianças, adolescentes, adultos e gestantes e estudos que focalizaram exclusivamente medidas psicofisiológicas, genéticas e de neuroimagem.

Com base nesses critérios, foram selecionados e analisados 68 artigos empíricos, procedendo-se, após a leitura dos mesmos, à categorização das informações quanto aos delineamentos adotados e às associações da depressão materna ao comportamento e à saúde mental dos filhos, analisando-se separadamente os estudos transversais e longitudinais, abordando também a associação da depressão materna a outras variáveis contextuais de risco.

\section{Principais caracteristicas dos delineamentos adota- dos}

Foram identificados, na totalidade, exclusivamente estudos com delineamento de pesquisa quantitativa, sendo 45 estudos prospectivos longitudinais $(66,2 \%)$ e 23 transversais $(33,8 \%)$.

No conjunto dos 68 estudos, destaca-se o predomínio da utilização de amostras da comunidade (51,5\%) e de amostras de conveniência (58,8\%), sendo que, da totalidade dos artigos analisados, 16 foram desenvolvidos com amostras selecionadas de modo aleatório e/ou randômico $(23,5 \%)$. Tais amostras incluíram um número diverso de participantes, que variou de 39 a 7866 crianças, com destaque para 25 artigos nos quais as amostras foram extraídas de estudos de coorte $(36,8 \%)$.

Em relação aos tipos de medidas utilizadas nos estudos analisados, constatou-se que todos os artigos utilizaram medidas de autorrelato, 20 utilizaram também medidas observacionais $(29,4 \%)$, por meio de filmagem em vídeo ou gravação da voz em situação natural e/ou em laboratório, e dois estudos utilizaram ainda medidas laboratoriais, psicofisiológicas $(2,9 \%)$.

Quanto às estratégias de avaliação, evidenciou-se variação no número de avaliações realizadas nos estudos longitudinais, sendo que a maioria realizou de quatro a seis avaliações $(35,6 \%)$. Em relação aos estudos transversais, em 10 artigos foram realizadas comparações entre grupos $(43,5 \%)$, outros oito realizaram comparações entre e intragrupos $(34,8 \%)$ e cinco deles realizaram comparações exclusivamente intragrupos, não adotando a comparação com grupos controle $(21,7 \%)$.

Verificou-se, no conjunto dos estudos, o predomínio da utilização de avaliação sistemática para a confirmação diagnóstica quanto aos indicadores de psicopatologia infantil, com destaque para a utilização do Child Behavior Checklist (CBCL) em 24 estudos (51,1\%), e para a confirmação da depressão materna, em 21 estudos (60\%) foi utilizada a Entrevista Clínica Estruturada para o DSM-IV (Structured Clinical Interview - SCID). Nos estudos que utilizaram instrumentos de rastreamento para a identificação de sinais e sintomas de depressão, predominou o uso do Beck Depression Inventory (BDI), utilizado em 15 estudos $(29,4 \%)$.

Para o rastreamento de indicadores de psicopatologia e 
problemas comportamentais das crianças, 11 estudos (16,2\%) utilizaram o Child Depression Inventory (CDI) para a avaliação de sintomas depressivos e seis estudos $(8,8 \%)$, o Questionário de Capacidades e Dificuldades (SDQ) para a identificação de características comportamentais.

No que diz respeito às fontes de informações, a maioria dos estudos utilizaram pelo menos dois informantes, sendo que em $58,8 \%$ dos estudos as informações sobre a psicopatologia infantil foram coletadas com pelo menos um dos pais e o filho. Alguns estudos utilizaram também como fonte de informação o professor e/ou outros familiares (27,9\%), o que, somado aos estudos com pelo menos dois informantes, aponta para a relevância dada à triangulação de fontes de informações quando da avaliação de condições contextuais de risco e de indicadores de saúde mental infantil.

Analisando-se as características dos delineamentos adotados pelos 68 estudos, constata-se a busca de rigor no controle metodológico, especialmente quando da inclusão de variáveis do contexto familiar, e, nos estudos de seguimento, destacamse a relevância dos estudos com amostras da comunidade, e procedentes de estudos de coorte. Tais aspectos metodológicos, e especialmente a inclusão das variáveis contextuais, colocaram em foco nas análises a diversidade de fatores e o seu peso diferenciado, ao longo do tempo, como elementos relevantes para a compreensão das trajetórias de desenvolvimento infantil.

A busca pela qualidade metodológica foi também observada em alguns estudos quanto à utilização de: (a) medidas observacionais, (b) pelo menos dois informantes, (c) confirmação diagnóstica (para mães e/ou crianças), e (d) nos estudos transversais, a comparação entre e intragrupos. Destacase, assim, a escolha por estratégias de investigação que visam minimizar vieses e variáveis de confundimento quanto aos dados.

\section{Associações da depressão materna com o comporta- mento e a saúde mental das crianças}

Tanto nos estudos transversais $(n=23)$ quanto nos longitudinais $(n=45)$, observou-se que a depressão materna influenciou o comportamento e a saúde mental infantil, o que foi verificado por meio de medidas específicas e de associações com variáveis contextuais diversas.

Nos estudos analisados, tanto nos transversais quanto nos longitudinais, predominou a presença de associações da depressão materna aos problemas comportamentais e de psicopatologia das crianças, como variáveis principais avaliadas para as crianças, com maior prejuízo quanto às dificuldades emocionais e comportamentais, as manifestações depressivas e os problemas de conduta. Verificou-se em três estudos peculiaridades quanto a tais associações, a saber: em dois estudos longitudinais, a depressão materna associada à expressão emocional crítica mostrou-se relacionada à psicopatologia das crianças (Silk, Shaw, Skuban, Oland, \& Kovacs, 2006) e a super utilização dos serviços de saúde mental (Boyd, Chen, \& Benton, 2009); e no estudo transversal correlacional de Ferriolli et al. (2007) as associações se fizeram presentes quanto ao estresse materno e à instabilidade financeira.

Apresentar-se-á inicialmente a análise dos artigos transversais e em seguida dos artigos longitudinais. A Tabela
1 apresenta a distribuição dos estudos transversais quanto à presença ou ausência das associações da depressão materna, tendo por referência os indicadores comportamentais e de psicopatologia infantil avaliados.

No conjunto dos artigos, verificou-se o predomínio de associações negativas da depressão materna isoladamente e em conjunto com outras condições contextuais a dificuldades comportamentais e de saúde mental das crianças avaliadas.

Dentre os estudos que avaliaram as dificuldades emocionais e comportamentais das crianças, em geral, evidenciou-se associações predominantemente com os sintomas internalizantes. Tal associação, contudo, foi identificada em cinco estudos, quando da presença de outras condições de risco, tais como: o estresse pré-natal (Rice et al., 2010), o estilo parental negativo (Dietz et al., 2008; Rice et al., 2010), a expressão emocional crítica das mães (Tompson et al., 2010), a comorbidade com outros transtornos mentais (Barnow, Spitzer, Grabe, Kessler, \& Freyberger, 2006), e o gênero feminino, o qual esteve associado a estratégias de regulação emocional mais passiva para as meninas (Silk et al., 2006).

O único estudo que avaliou manifestações depressivas das crianças e não identificou impacto negativo constatou correlação entre a depressão materna e problemas de conduta das crianças, em especial para os meninos em idade escolar e para crianças expostas a práticas parentais inadequadas (Drabick et al., 2006), sendo destacada a influência das variáveis gênero e ambiente familiar para problemas de comportamento antissocial. O comportamento parental negativo também foi uma condição contextual identificada como mediadora de problemas de conduta (Chen \& Johnston, 2007) e de comportamentos externalizantes, principalmente diante de maior gravidade e cronicidade da depressão materna (Foster, Garber, \& Durlak, 2008).

Quanto aos demais indicadores de problemas comportamentais externalizantes, destacam-se que nos quatro estudos que avaliaram hiperatividade e problemas de atenção não foram identificadas associações da depressão materna a tais dificuldades (Bergeron et al., 2007; Drabick et al., 2006; Kopp \& Beauchaine, 2007; Rice et al., 2010), o que foi observado também em dois dos três estudos que avaliaram o comportamento desafiador opositor (Bergeron et al., 2007; Kopp \& Beauchaine, 2007) e em dois dos seis estudos que avaliaram problemas de conduta (Bergeron et al., 2007; Rice et al., 2010).

Outros dois estudos transversais constataram associações da depressão materna com prejuízos na socialização da criança no ambiente familiar (Gregory et al., 2012) e com dificuldades de relacionamento com os pares (Kersten-Alvarez et al., 2012).

Verificou-se na análise da literatura, de forma consistente, a correlação entre a depressão materna e os indicadores de prejuízos à saúde mental infantil, com o predomínio de estudos que avaliaram problemas emocionais e comportamentais em geral e manifestações depressivas próprias dos comportamentos internalizantes, sinalizando associações evidentes da depressão materna a tais manifestações, por outro lado não foi verificada tal evidência quanto aos comportamentos externalizantes.

As associações entre a depressão materna e problemas emocionais e comportamentais e de saúde mental infantil foram constatadas em todos os estudos longitudinais $(n=45)$, 
Tabela 1

Estudos Transversais: Associações da Depressão Materna (D.M.) com Indicadores Comportamentais e de Psicopatologia das Crianças $(n=23) *$

\begin{tabular}{lll}
\hline $\begin{array}{c}\text { Indicadores comportamentais e de psicopatologia avaliados } \\
\text { (Número de estudos) }\end{array}$ & $\begin{array}{c}\text { Associações } \\
\text { da D.M. }\end{array}$ & $\begin{array}{c}\text { Estudos } \\
\text { (Frequência) }\end{array}$ \\
\hline Dificuldades emocionais e comportamentais $(\boldsymbol{n = 1 4 )}$ & Presente & $\begin{array}{l}06,21,22,28,29,31,37,41,50,55,56,59, \\
64,66(\mathbf{1 4})\end{array}$ \\
Manifestações depressivas $(\boldsymbol{n}=\mathbf{0 8})$ & Presente & $07,26,38,42,43,56,64(\mathbf{0 7})$ \\
& Ausente & $22(\mathbf{0 1})$ \\
Manifestações de ansiedade $(\boldsymbol{n}=\mathbf{0 4})$ & Presente & $26,64(\mathbf{0 2})$ \\
& Ausente & $07,38(\mathbf{0 2})$ \\
Problemas de conduta e comportamentos antissociais $(\boldsymbol{n}=\mathbf{0 7})$ & Presente & $16,22,26,43,64(\mathbf{0 5})$ \\
& Ausente & $07,55(\mathbf{0 2})$ \\
Comportamento desafiador opositivo $(\boldsymbol{n}=\mathbf{0 3})$ & Presente & $64(\mathbf{0 1})$ \\
Hiperatividade e problemas de atenção $(\boldsymbol{n}=\mathbf{0 4})$ & Ausente & $07,43 \mathbf{( 0 2 )}$ \\
Déficits cognitivos e verbais $(\boldsymbol{n}=\mathbf{0 3})$ & Ausente & $07,22,43,55 \mathbf{( 0 4 )}$ \\
Prejuízo no funcionamento global adaptativo $(\boldsymbol{n}=\mathbf{0 1})$ & Presente & $20,38,40 \mathbf{( 0 3 )}$ \\
Maior reatividade emocional $(\boldsymbol{n}=\mathbf{0 1})$ & Presente & $26(\mathbf{0 1})$ \\
Prejuízo na interação mãe-criança $(\boldsymbol{n}=\mathbf{0 1})$ & Presente & $37(\mathbf{0 1})$ \\
\hline Outros $(\boldsymbol{n}=\mathbf{0 2})$ & Presente & $21(\mathbf{0 1})$ \\
\hline
\end{tabular}

Nota. *11 artigos incluídos em mais de uma categoria.

06 Barnow, Spitzer, Grabe, Kessler e Freyberger (2006); 07 Bergeron et al. (2007); 16 Chen e Johnston (2007); 20 Dearing e Gotlib (2009); 21 Dietz et al. (2008); 22 Drabick, Beauchaine, Gadow, Carlson e Bromet (2006); 26 Feder et al. (2008); 28 Ferrioli et al. (2007); 29 Foster, Garber e Durlak (2008); 31 Green, Stanley e Peters (2007); 32 Gregory et al. (2012); 37 Jaser et al. (2008); 38 Joorman, Talbot e Gotlib (2007); 40 Kersten-Alvarez et al. (2012); 41 Kim-Cohen, Caspi, Rutter, Tomás e Moffitt (2006); 42 Kiss et al. (2007); 43 Kopp e Beauchaine (2007); 50 Pachter, Auinger, Palmer e Weitzman (2006); 55 Rice et al. (2010); 56 Shannon, Beauchaine, Brenner, Neuhaus e Gatzke-Kopp (2007); 59 Silk, Shaw, Skuban, Oland e Kovacs (2006); 63 Tompson et al. (2010); 65 Vasa et al. (2007).

conforme apresentação na Tabela 2. Destaca-se que as crianças foram avaliadas em diferentes etapas do desenvolvimento, sendo referidas nessa análise as associações com os problemas de saúde mental na idade escolar, como objetivo proposto para o estudo.

Verificou-se que três estudos avaliaram exclusivamente crianças no período escolar (Barker, Copeland, Maughan, Jaffee, \& Uher, 2012; Loeber, Hipwell, Battista, Sembower, \& Stouthamer-Loeber, 2009; van der Molen, Hipwell, Vermeiren, \& Loeber, 2011) e 12 estudos avaliaram o impacto também para crianças antes deste período (26,7\%). Dos 45 artigos com delineamento longitudinal, 13 estudos $(28,9 \%)$ estenderam as avaliações da pré-escola até a adolescência, outros $11(24,4 \%)$ da fase escolar à adolescência e quatro $(8,9 \%)$, da fase escolar até a fase adulta. Destaca-se que dois estudos longitudinais tinham um enfoque multigeracional, tendo realizado avaliações com três gerações (filhos, pais e avós com depressão), sendo que esses verificaram a continuidade intergeracional da depressão (Grillon et al., 2005; Warner, Wickramaratne, \& Weissman, 2008), enfatizando o peso de fatores genéticos associados aos ambientais na transmissão deste transtorno.

Dentre as dificuldades emocionais e comportamentais em geral, o impacto para os sintomas internalizantes (Barker et al., 2012; Chronis-Tuscano et al., 2010; Elgar, Mills, McGrath, Waschbusch, \& Brownridge, 2007; Pilowsky, Wickramaratne, Nomura, \& Weissman, 2006; Shaw, Hyde, \& Brennan, 2012;
Warner et al., 2008) também foi o mais avaliado entre os estudos de seguimento. Tal associação foi ainda identificada especificamente para sintomas depressivos e ansiosos em outros 13 estudos longitudinais, dos quais nove $(69,2 \%)$ constataram impacto negativo da depressão materna, independente de sua associação a outras condições contextuais.

Dentre os estudos longitudinais que estudaram especificamente o comportamento externalizante, dois identificaram associações com a depressão materna para préescolares e escolares (Galéra et al., 2011; Shaw, Lacourse, \& Nagin, 2005), quatro exclusivamente para escolares (Barker et al., 2012; Loeber et al., 2009; Pawlby, Sharp, Hay, \& O'Keane, 2008; van der Molen et al., 2011), e três para escolares e adolescentes (Hay, Pawlby, Waters, \& Sharp, 2008; Pilowsky et al., 2008; Shaw et al., 2012). Foram ainda observadas associações com dificuldades diversas em estudos que avaliaram o comportamento geral dos filhos, tais como: manifestações de agressividade em pré-escolares e escolares (Gross, Shaw, \& Moilanen, 2008) e em escolares e adolescentes (Elgar et al. 2007), comportamento antissocial em escolares e adolescentes (Gross et al, 2008; Shaw et al., 2012), problemas de conduta de pré-escolares a adolescentes (Barker \& Maughan, 2009), comportamento desafiador opositor em escolares e adolescentes (Cortes, Fleming, Mason, \& Catalano 2009) e o abuso de substâncias psicoativas em adolescentes (Cortes et al., 2009; 
Tabela 2

Estudos Longitudinais: Associações da Depressão Materna (D.M.) com Indicadores Comportamentais e de Psicopatologia das Crianças $(\mathrm{n}=45)^{*}$

\begin{tabular}{|c|c|c|}
\hline $\begin{array}{c}\text { Indicadores comportamentais e de psicopatologia } \\
\text { avaliados } \\
\text { (Número de estudos) }\end{array}$ & $\begin{array}{l}\text { Associações da } \\
\text { D.M. }\end{array}$ & $\begin{array}{c}\text { Estudos } \\
\text { (Frequência) }\end{array}$ \\
\hline Dificuldades emocionais e comportamentais $(\boldsymbol{n}=\mathbf{2 4})$ & Presente & $\begin{array}{l}02,03,05,08,12,13,15,19,23,24,25,34,35,45,49, \\
53,57,58,60,61,62,66,67,68 \text { (24) }\end{array}$ \\
\hline Manifestações depressivas $(\boldsymbol{n}=\mathbf{1 7})$ & Presente & $\begin{array}{l}01,04,09,11,14,17,18,27,36,39,44,46,47,48, \\
52,54,58(\mathbf{1 7})\end{array}$ \\
\hline \multirow{2}{*}{ Manifestações de ansiedade $(\boldsymbol{n}=\mathbf{1 3})$} & Presente & $04,09,18,27,36,39,44,46,51,52,54,58$ (12) \\
\hline & Ausente & $47(01)$ \\
\hline \multirow{2}{*}{$\begin{array}{l}\text { Problemas de conduta e comportamento antissocial ( } \boldsymbol{n} \\
=\mathbf{0 9})\end{array}$} & Presente & $04,05,34,35,46,54,58,64$ (08) \\
\hline & Ausente & $09(\mathbf{0 1})$ \\
\hline \multirow{2}{*}{ Comportamento desafiador opositivo $(\boldsymbol{n}=\mathbf{0 5})$} & Presente & $04,52,58,64(04)$ \\
\hline & Ausente & $09(01)$ \\
\hline \multirow{2}{*}{ Hiperatividade e problemas de atenção $(\boldsymbol{n}=\mathbf{0 6})$} & Presente & $04,30,46,52,58(\mathbf{0 5})$ \\
\hline & Ausente & $09(\mathbf{0 1})$ \\
\hline Déficits cognitivos e verbais $(\boldsymbol{n}=\mathbf{0 3})$ & Presente & $14,24,35(\mathbf{0 3})$ \\
\hline Prejuízo no funcionamento global adaptativo $(\boldsymbol{n}=\mathbf{0 4})$ & Presente & $54,62,67,68(04)$ \\
\hline Maior utilização de serviços de saúde mental $(\boldsymbol{n}=\mathbf{0 2})$ & Presente & $10,67(\mathbf{0 2})$ \\
\hline Maior reatividade emocional $(\boldsymbol{n}=\mathbf{0 1})$ & Presente & $33(01)$ \\
\hline Autoimagem negativa $(\boldsymbol{n}=\mathbf{0 1})$ & Presente & $01(01)$ \\
\hline Outros $(\boldsymbol{n}=\mathbf{0 2})$ & Presente & $17,14(\mathbf{0 2})$ \\
\hline
\end{tabular}

Nota. $* 21$ artigos incluídos em mais de uma categoria.

01 Abela, Morrison e Starrs (2007); 02 Asbury, Dunn e Plomin (2006); 03 Bagner, Pettit, Lewinsohn e Seeley (2010); 04 Barker, Copeland, Maughan, Jaffee e Uher (2012); 05 Barker e Maughan (2009); 08 Blatt-Eisengart, Drabick, Monahan e Steinberg (2009); 09 Boyce et al. (2006); 10 Boyd, Chen e Benton (2009); 11 Bureau, Easterbrooks e Lyons-Ruth (2009); 12 Burt et al. (2005); 13 Campbell, Matestic, Stauffenberg, Mohan e Kirchner (2007); 14 Carter e Garber (2011); 15 Chang, Halpernm e Kaufman (2007); 17 Chronis-Tuscano et al. (2010); 18 Corapci, Smith e Lozoff (2006); 19 Cortes, Fleming, Mason e Catalano (2009); 23 Elgar, Mills, McGrath, Waschbusch e Brownridge (2007); 24 Essex et al. (2006); 25 Fanti e Henrich (2010); 27 Feng, Shaw e Silk (2008); 30 Galéra et al. (2011); 33 Grillon et al. (2005); 34 Gross, Shaw e Moilanen (2008); 35 Hay, Pawlby, Waters e Sharp (2008); 36 Jacobs, Miller, Wickramaratne, Gameroff, e Weissman (2012); 39 Karevold, Roysam, Ystrom e Mathiesen (2009); 44 Leech, Larkby, Day e Day (2006); 45 Lengua, Bush, Long, Kovacs e Trancik (2008); 46 Loeber, Hipwell, Battista, Sembower e Stouthamer-Loeber (2009); 47 Milan, Snow e Belay (2009); 48 Monshouwer et al. (2012); 49 Olino et al. (2011); 51 Pagani, Japel, Vaillancourt, Côté \& Tremblay (2007); 52 Pawlby, Sharp, Hay e O'Keane (2008); 53 Pilowsky, Wickramaratne, Nomura e Weissman (2006); 54 Pilowsky et al. (2008); 57 Shaw, Lacourse e Nagin (2005); 58 Shaw, Hyde e Brennan (2012); 60 Sourander et al. (2006); 61 Sterba, Prinstein e Cox (2007); 62 Talati et al. (2007); 64 van der Molen, Hipwell, Vermeiren, e Loeber (2011); 66 Warner, Wickramaratne e Weissman (2008); 67 Weissman et al. (2006); 68 Wickramaratne et al. (2011).

Weissman et al., 2006).

Um único estudo não identificou correlação estatisticamente significativa entre depressão materna e comportamentos externalizantes de escolares (Boyce et al., 2006), contudo, constatou a presença de mais problemas de comportamento internalizante quando das associações com baixo envolvimento paterno na infância, com maior gravidade dos sintomas de saúde mental em geral e para o gênero feminino.

Tais achados evidenciaram a ênfase na investigação de comportamentos externalizantes predominantemente a partir da idade escolar. A escolha pela avaliação de indicadores de comportamentos internalizantes e externalizantes pode ser observada, por exemplo, no estudo de Asbury, Dunn e Plomin (2006) com crianças gêmeas, o qual identificou a presença de indicadores de hiperatividade, problemas de atenção, problemas de conduta e no relacionamento com os pares, bem como problemas de comportamento internalizante, para escolares, quando da presença de variáveis contextuais relacionadas às interações negativas entre pais e filhos.

Com relação a outras variáveis estudadas, destacam-se a verificação da associação da depressão materna ao estresse como potencializadoras da relação dos problemas de saúde mental em adolescentes (Carter \& Garber, 2011), assim como a verificação de problemas emocionais aos 4 e 6 anos por parte das crianças com maior risco de suicídio na adolescência (Chronis-Tuscano et al., 2010), e a convivência com a depressão materna no primeiro ano de vida da criança identificada como uma condição de maior vulnerabilidade para problemas futuros de saúde mental infantil (Bagner, Pettit, Lewinsohn, \& Seeley, 2010).

Condições adversas do ambiente familiar foram associadas 
à depressão materna influenciando as trajetórias de problemas das crianças ao longo do período pré-escolar e escolar, como verificado nos estudos de Fanti e Henrich (2010) quanto aos riscos do ambiente familiar e a co-ocorrência de problemas externalizantes e internalizantes; de Feng et al. (2008) quando da avaliação do controle parental negativo; de Talati et al. (2007) com famílias monoparentais e de Pagani, Japel, Vaillancourt, Côté e Tremblay (2008) com relação às variáveis, baixo nível sócio econômico, conflitos familiares e gênero feminino.

Todos os 68 artigos analisados incluíram em suas avaliações, além da depressão materna, outras variáveis contextuais, buscando verificar se essas estavam associadas às dificuldades comportamentais e de saúde mental das crianças. Tais variáveis referem-se a características sociodemográficas, aspectos clínicos do transtorno materno, e peculiaridades do ambiente familiar quanto às interações e às práticas educativas. Tendo em vista a escolha das palavras-chave para a busca de artigos, identificou-se um número maior de condições potencializadoras das dificuldades.

Algumas condições avaliadas foram identificadas como favorecedoras de comportamentos e condições de saúde mental positiva das crianças, sendo tais variáveis: o partilhar pela mesma religião pelos membros da família (Jacobs, Miller, Wickramaratne, Gameroff, \& Weissman, 2012); o gênero feminino para a presença de mais comportamento pró-social (Essex et al., 2006), bons recursos verbais das próprias crianças (Corapci, Smith, \& Lozoff, 2006), as características de menor gravidade do transtorno depressivo das mães (Campbell, Matestic, Von Stauffenber, Mohan, \& Kirchner, 2007; Pilowsky et al., 2008); as práticas educativas, com cuidado parental adequado (Elgar et al., 2007), a presença paterna no cotidiano da criança (Sterba, Prinstein, \& Cox, 2007) e o comportamento afetivo e responsivo da mãe frente à criança (Foster et al., 2008).

A variável gênero, nos estudos longitudinais, pode ser destacada como a mais relacionada a problemas de saúde mental para as crianças. Foi verificado que as meninas que convivem com a depressão materna apresentaram mais problemas em todas as idades de desenvolvimento estudadas (Elgar et al., 2007; Hay et al., 2008; Kersten-Alvarez et al., 2012; Kiss et al., 2007; Shannon, Beauchaine, Brenner, Neuhaus, \& Kopp, 2007; Sourander et al., 2006; Sterba et al., 2007) e com relação aos meninos em idade escolar, foi verificada a presença de mais problemas de conduta (Lengua, Bush, Long, Kovacs, \& Trancik, 2008), de menos comportamento pró-social (Essex et al., 2006) e de mais sintomas internalizantes (Karevold, Roysamb, Ystrom, \& Mathiesen, 2009).

Quanto às características clínicas da depressão das mães, verificou-se que a maior gravidade e o maior tempo de exposição da criança à sintomatologia materna foram associados a mais problemas emocionais e comportamentais e mais prejuízo no funcionamento global por parte de escolares e adolescentes (Wickramaratne et al., 2011); e, ainda, que a presença de comorbidades com outros transtornos mentais foi associada a mais sintomas depressivos (Hay et al., 2008) e mais manifestações emocionais e comportamentais em geral para crianças em idade escolar (Karevold et al., 2009; Loeber et al., 2009) e em diferentes etapas do desenvolvimento (Barker
\& Maughan, 2009; Campbell et al., 2007; Gross et al., 2008; Sterba et al., 2007).

A depressão materna foi associada ainda à presença de mais problemas emocionais e comportamentais quando da presença de interações familiares pobres e negativas, com destaque para a identificação de: apego infantil inseguro (Milan, Snow, \& Belay, 2009), conflitos familiares recorrentes (Burt et al., 2005; Pilowsky et al., 2006; Sourander et al., 2006), famílias monoparentais ou com ausência do pai (Chang, Halpernm, \& Kaufman 2007), e práticas parentais inadequadas, tais como práticas educativas com punição severa (Barker \& Maughan, 2009; Loeber et al., 2009), estilo parental inadequado (Bureau, Easterbrooks, \& Lyons-Ruth, 2009; Pilowsky et al., 2006), rejeição e comportamento negativo (Elgar et al., 2007).

No conjunto de estudos identificados, a análise das associações entre depressão materna e aspectos comportamentais e de saúde mental das crianças evidenciou que a convivência com tal adversidade mostrou-se associada, predominantemente, à presença de dificuldades emocionais e comportamentais em geral, de manifestações depressivas e de ansiedade. Destacase, assim, com base nessa revisão sistemática da literatura, que a depressão materna se configura como uma condição de risco ao desenvolvimento infantil nas diferentes etapas do desenvolvimento, se associando predominantemente aos sintomas internalizantes para as meninas, e aos para sintomas externalizantes, quando da avaliação de escolares e adolescentes.

Características do ambiente familiar, tais como estilo parental, organização familiar e interações intrafamiliares, também se configuraram como variáveis associadas à depressão materna relevantes para a análise das condições contextuais adversas, concorrendo para as dificuldades comportamentais e de psicopatologia verificadas. Destaca-se que tais variáveis foram abordadas em análises multivariadas, sinalizando a necessidade de compreensão dos múltiplos fatores que concorrem para os processos de adaptação das crianças frente às condições adversas.

\section{Considerações finais}

Evidenciou-se nos 68 artigos analisados bons controles metodológicos, explícitos pela ênfase nos estudos de seguimento, com confirmação diagnóstica para mães e/ou crianças e triangulação de fontes de informações. Contudo, verificou-se como pontos críticos: pouca informação sobre as características clínicas da depressão materna, tais como recorrência, gravidade e tempo e período de exposição da criança à sintomatologia das mães; bem como o uso de uma diversidade de instrumentos para a avaliação dos indicadores de dificuldades comportamentais e de psicopatologia infantil, e a inclusão de um extenso número de variáveis como condições contextuais, além de distintos critérios de inclusão e exclusão dos participantes, aspectos estes que dificultaram as comparações dos dados de forma mais específica.

Observou-se ainda, como limites presentes em alguns estudos específicos: a utilização de amostras de conveniência e muito peculiares, tais como comparações entre raças, o que limita a generalização dos resultados, além da falta de informações a respeito da psicopatologia paterna na maioria dos estudos e a não consideração do risco genético para a continuidade 
intergeracional da depressão, a qual foi avaliada em apenas dois estudos.

Considerando que a depressão materna, pelas suas peculiaridades de recorrência e cronicidade, é uma condição que expõe as crianças a múltiplas situações adversas; tais limites sugerem a necessidade de novos estudos que focalizem, além da depressão materna, variáveis contextuais, avaliadas por instrumentos que permitam maior comparação entre os dados, e que inclusive combinem medidas biológicas e psicossociais para a verificação da continuidade intergeracional dos sintomas depressivos. Destaca-se, nesse contexto, a relevância de se compreender, além das condições que aumentam os riscos de dificuldades, as variáveis que favorecem as ações protetoras para a saúde mental de crianças que convivem com a depressão materna, com ênfase nos padrões comportamentais internalizantes e externalizantes, a fim de reunir indicadores que favoreçam o planejamento de intervenções junto a famílias que convivem com a depressão.

\section{Referências}

Abela, J. R. Z., Morrison, E., \& Starrs, C. (2007). Excessive reassurance seeking, self-esteem, and depressive symptoms in children of affectively ill parents: An experience sampling analysis. Journal of Social and Clinical Psychology, 26(7), 849-869.

Andrade, L. H. S. G., Viana, M. C., \& Silveira, C. M. (2006). Epidemiologia dos transtornos psiquiátricos na mulher. Revista de Psiquiatria Clínica, 33(2), 43-54.

Asbury, K., Dunn, J. F., \& Plomin, R. (2006). Birthweight-discordance and differences in early parenting relate to monozygotic twin differences in behavior problems and academic achievement at age 7. Developmental Science, 9(2), 22-31.

Bagner, D. M., Pettit, J. W., Lewinsohn, P. M., \& Seeley, J. R. (2010). Effect of maternal depression on child behavior: a sensitive period? Journal of the American Academy of Child \& Adolescent Psychiatry, 49(7), 699-707.

Barker, E. D., Copeland, W., Maughan, B., Jaffee, S. R., \& Uher, R. (2012). Relative impact of maternal depression and associated risk factors on offspring psychopathology. The British Journal of Psychiatry, 200(2), 124-129.

Barker, E. D., \& Maughan, B. (2009). Differentiating early-onset persistent versus childhood-limited conduct problem. The American Journal of Psychiatry, 166(8), 900-908.

Barnow, S., Spitzer, C., Grabe, H. J., Kessler, C., \& Freyberger, H. J. (2006). Individual characteristics, familial experience, and psychopathology in children of mothers with borderline personality disorder. Journal of the American Academy of Child \& Adolescent Psychiatry, 45(8), 965-972.

Belfer, M. L. (2008). Child and adolescent mental disorders: The magnitude of the problem across the globe. Journal of Child Psychology and Psychiatry, 49(3), 226-236. doi: 10.1111/j.1469-7610.2007.01855.x.

Bergeron, L., Valla, J. P., Smolla, N., Piché, G., Berthiaume, C., \& St-Georges, M. (2007). Correlates of depressive disorders in the Quebec general population 6 to 14 years of age. Journal of Abnormal Child Psychology, 35, 459-474.

Blatt-Eisengart, I., Drabick, D. A. G., Monahan, K. C., \& Steinberg, L. (2009). Sex differences in the longitudinal relations among family risk factors and childhood externalizing symptoms. Developmental Psychology, 45(2), 491-502.

Boyce, W. T., Essex, M. J., Alkon, A., Goldsmith, H. H., Kraemer, H. C., \& Kupfer, D. J. (2006). Early father involvement moderates biobehavioral susceptibility to mental health problems in middle childhood. Journal of the American Academy of Child \& Adolescent Psychiatry, 45(12), 1510-1520.
Boyd, R. C., Chen, E., \& Benton, T. (2009). A pilot investigation of the rates and predictors of mental health services utilization among depressed African American mothers and their children. Journal of the National Medical Association, 101(11), 1096-1103.

Bureau, J. F., Easterbrooks, M. A., \& Lyons-Ruth, K. (2009). Maternal depressive symptoms in infancy: Unique contribution to children's depressive symptoms in childhood and adolescence? Development Psychopathology, 21(2), 519-537.

Burt, K. B., Van Dulmen, M. H. M., Carlivati, J., Egeland, B., Sroufe, L. A., Forman, D. R., ... Carlson, E. A. (2005). Mediating links between maternal depression and offspring psychopathology: The importance of independent data. Journal of Child Psychology and Psychiatry, 46(5), 490-499. doi: 10.1111/j.1469-7610.2004.00367

Campbell, S. B., Matestic, P., Von Stauffenber, C., Mohan, R., \& Kirchner, T. (2007). Trajectories of maternal depressive symptoms, maternal sensitivity, and children's functioning at school entry. Developmental Psychology, 43(5), 1202-1215.

Carter, J. S., \& Garber, J. (2011). Predictors of the first onset of a major depressive episode and changes in depressive symptoms across adolescence: Stress and negative cognitions. Journal of Abnormal Psychology, 120(4), 779-796.

Chang, J. J, Halpernm, C. T., \& Kaufman, J. S. (2007). Maternal depressive symptoms, father's involvement, and the trajectories of child problem behaviors in a US national sample. Archives of Pediatrics \& Adolescent Medicine, 161(7), 697-703.

Chen, M., \& Johnston, C. (2007). Maternal inattention and impulsivity and parenting behaviors. Journal of Clinical Child and Adolescent Psychology, 36(3), 455-468.

Chronis-Tuscano, A., Molina, B. S., Pelham, W. E., Applegate, B., Dahlke, A., Overmyer, M., \& Lahey, B. B., (2010). Very early predictors of adolescent depression and suicide attempts in children with attention-deficit/ hyperactivity disorder. Archives of General Psychiatry, 67(10), 1044-1051. doi: 10.1001/archgenpsychiatry.2010.127

Corapci, F., Smith, J., \& Lozoff, B. (2006). The role of verbal competence and multiple risk on the internalizing behavior problems of Costa Rican youth. Annals of the New York Academy Sciences, 1094, 278-281.

Cortes, R. C., Fleming, C. B., Mason, W. A., \& Catalano, R. F. (2009). Risk factors linking maternal depressed mood to growth in adolescent substance use. Journal of Emotional and Behavioral Disorders, 17(1), 49-64.

Dearing, K. F., \& Gotlib, I. H. (2009). Interpretation of ambiguous information in girls at risk for depression. Journal of Abnormal Child Psychology, 37, 79-91.

Dietz, L. J., Birmaher, B., Williamson, D. E., Silk, J. S., Dahl, R. E., Axelson, D. A., ... Ryan, N. D. (2008). Mother-child interactions in depressed children and children at high risk and low risk for future depression. Journal of the American Academy Child \& Adolescent Psychiatry, 47(5), 574-82. doi: 10.1097/CHI.0b013e3181676595

Drabick, D. A. G., Beauchaine, T. P., Gadow, K. D., Carlson, G. A., \& Bromet, E. J. (2006). Risk factors for conduct problems and depressive symptoms in a cohort of ukrainian children. Journal of Clinical Child \& Adolescent Psychology, 35(2), 244-252.

Elgar, F. J., Mills, R. S., McGrath, P. J., Waschbusch, D. A., \& Brownridge, D. A. (2007). Maternal and paternal depressive symptoms and child maladjustment: The mediating role of parental behavior. Journal of Abnormal Child Psychology, 35(6), 943-955.

Essex, M. J., Kraemer, H. C., Armstrong, J. M., Boyce, W. T., Goldsmith, H. H., Klein, M. H., ... Kupfer, M. D. (2006). Exploring risk factors for the emergence of children's mental health problems. Archives of General Psychiatry, 63, 1246-1256. doi: 10.1001/archpsyc.63.11.1246

Fanti, K. A., \& Henrich, C. C. (2010). Trajectories of pure and co-occurring internalizing and externalizing problems from age 2 to age 12: Findings from the National Institute of Child Health and Human Development Study of Early Child Care. Developmental Psychology, 46(5), 1159-1175.

Feder, A., Alonso, A., Tang, M., Liriano, W., Warner, V., Pilowsky, D., \& 
Weismann, M. M. (2008). Children of low-income depressed mothers: Psychiatric disorders and social adjustment. Depression and Anxiety, 0, 1-8. doi: $10.1002 /$ da. 20522

Feng, X., Shaw, D. S., \& Silk, J. S. (2008). Developmental trajectories of anxiety symptoms among boys across early and middle childhood. Journal of Abnormal Psychology, 117(1), 32-47.

Ferriolli, S. H. T., Marturano, E. M., \& Puntel, L. P. (2007). Contexto familiar e problemas de saúde mental infantil no Programa Saúde da Família. Revista de Saúde Pública, 41(2), 251-259.

Foster, C. J. E., Garber, J., \& Durlak, J. A. (2008). Current and past maternal depression, maternal interaction behaviors, and children 's externalizing and internalizing symptoms. Journal of Abnormal Child Psychology, 36, 527-537.

Galéra, C., Côté, S. M., Bouvard, M. P., Pingault, J. B., Melchior, M., Michel, G., ... Tremblay, R. E. (2011). Early risk factors for hyperactivity-impulsivity and inattention trajectories from age 17 months to 8 years. Archives of General Psychiatry, 68(12), 1267-1275. doi: 10.1001/archgenpsychiatry.2011.138

Green, J., Stanley, C., \& Peters, S. (2007). Disorganized attachment representation and atypical parenting in young school age children with externalizing disorder. Attachment \& Human Development, 9(3), 207-222.

Gregory, A. M., Moffitt, T. E., Ambler, A., Arseneault, L., Houts, R. M., \& Caspi, A. (2012). Maternal insomnia and children's family socialization environments. Sleep, 35(4), 579-582.

Grillon, C., Warner, V., Hille, J., Merikangas, K. R., Bruder, G. E., Tenke, C. E., ... Weissman, M. M. (2005). Families at high and low risk for depression: A three-generation startle study. Biological Psychiatry, 57, 953-960. doi: 10.1016/j.biopsych.2005.01.045

Gross, H. E., Shaw, D. S., \& Moilanen, K. L. (2008). Reciprocal associations between boys' externalizing problems and mothers' depressive symptoms. Journal of Abnormal Child Psychology, 36(5), 693-709.

Halpern, R., \& Figueiras, A. C. M. (2004). Influências ambientais na saúde mental da criança. Jornal de Pediatria, 80(2), S104-S110.

Hay, D. F., Pawlby, S., Waters, C. S., \& Sharp, D. (2008). Antepartum and postpartum exposure to maternal depression: different effects on different adolescent outcomes. Journal of Child Psychology and Psychiatry, 49(10), 1079-1088.

Jacobs, M., Miller, L., Wickramaratne, P., Gameroff, M., \& Weissman, M. M. (2012). Family religion and psychopathology in children of depressed mothers: ten-year follow-up. Journal of Affective Disorders, 136(3), 320-327.

Jaser, S. S., Fear, J. M., Reeslund, K. L., Champion, J. E., Reising, M. M., \& Compas, B. E. (2008). Maternal sadness and adolescents responses to stress in offspring of mothers with and without a history of depression. Journal of Clinical Child \& Adolescent Psychology, 37(4), 736-746.

Joormann, J., Talbot, L., \& Gotlib, I. H. (2007). Biased processing of emotional information in girls at risk for depression. Journal of Abnormal Psychology, 116(1), 135-143

Karevold, E., Roysamb, E., Ystrom, E., \& Mathiesen, K. S. (2009). Predictors and pathways from infancy to symptoms of anxiety and depression in early adolescence. Developmental Psychology, 45(4), 1051-1060.

Kersten-Alvarez, L. E., Hosman, C. M., Riksen-Walraven, J. M., van Doesum, K. T., Smeekens, S., \& Hoefnagels, C. (2012). Early school outcomes for children of postpartum depressed mothers: Comparison with a community sample. Child Psychiatry \& Human Development, 43(2), 201-218.

Kim-Cohen, J., Caspi, A., Rutter, M., Tomás, M. P., \& Moffitt, T. E. (2006). The caregiving environments provided to children by depressed mothers with or without an antisocial history. American Journal of Psychiatry, 163 , 1009-1018.

Kiss, E., Gentzler, A. M., George, C., Kapornai, K., Tamás, Z., Kovacs, M., \& Vetró, A. (2007). Factors influencing mother-child reports of depressive symptoms and agreement among clinically referred depressed youngsters in Hungary. Journal of Affective Disorders, 100, 143-151. doi: 10.1016/j. jad.2006.10.008
Kopp, L. M., \& Beauchaine, T. P. (2007). Patterns of psychopathology in the families of children with conduct problems, depression, and both psychiatric conditions. Journal of Abnormal Child Psychology, 35(2), 301-312.

Leech, S. L., Larkby, C. A., Day, R., \& Day, N. L. (2006). Predictors and correlates of high levels of depression and anxiety symptoms among children at Age 10. Journal of American Academy of Child \& Adolescent Psychiatry, 45(2), 223-230.

Lengua, L. J., Bush, N. R., Long, A. C., Kovacs, E. A., \& Trancik, A. M. (2008). Effortful control as a moderator of the relation between contextual risk factors and growth in adjustment problems. Development and Psychopathology, 20, 509-528.

Loeber, R., Hipwell, A., Battista, D., Sembower, M., \& Stouthamer-Loeber, M. (2009). Intergenerational transmission of multiple problem behaviors: prospective relationships between mothers and daughters. Journal of Abnormal Child Psychology, 37, 1035-1048.

Markowitz, J. C. (2008). Depressed mothers, depressed children. American Journal of Psychiatry, 165, 1086-1088.

Milan, S., Snow, S., \& Belay, S. (2009). Depressive symptoms in mothers and children: Preschool attachment as a moderator of risk. Developmental Psychology, 45(4), 1019-1033.

Monshouwer, K., Smit, F., Ruiter, M., Ormel, H., Verhulst, F., Vollebergh, W., \& Oldehinkel, T. (2012). Identifying target groups for the prevention of depression in early adolescence: The TRAILS study. Journal of Affectives Disorders, 138(3), 287-294. doi: 10.1016/j.jad.2012.01.026

Olino, T. M., Lopez-Duran, N. L., Kovacs, M., George, C. J., Gentzler, A. L., \& Shaw, D. S. (2011). Developmental trajectories of positive and negative affect in children at high and low familial risk for depressive disorder. Journal of Child Psychology and Psychiatry, 52(7), 792-799.

Pachter, L. M., Auinger, P., Palmer, R., \& Weitzman, M. (2006). Do parenting and the home environment, maternal depression, neighborhood, and chronic poverty affect child behavioral: Problems differently in different racial-ethnic groups? Pediatrics, 117(4), 1329-1338.

Pagani, L. S., Japel, C., Vaillancourt, T., Côté, S., \& Tremblay, R. E. (2008). Links between life course trajectories of family dysfunction and anxiety during middle childhood. Journal of Abnormal Child Psychology, 36, 41-53.

Pawlby, S., Sharp, D., Hay, D., \& O'Keane, V. (2008). Postnatal depression and child outcome at 11 years: The importance of accurate diagnosis. Journal of Affective Disorders, 107, 241-245.

Pilowsky, D. J., Wickramaratne, P., Nomura, Y., \& Weissman, M. M. (2006). Family discord, parental depression, and psychopathology in offspring: 20-year follow-up. Journal of American Academy Child \& Adolescent Psychiatry, 45(4), 452-460.

Pilowsky, D. J., Wickramaratne, P., Talati, A., Tang, M., Hughes, C. W., Garber, J., .. Weissman, M.M. (2008). Children of depressed mothers 1 year after the initiation of maternal treatment: Findings from the STAR*D-child study. American Journal of Psychiatry, 165(9), 1136-1147. doi: 10.1176/appi. ajp.2008.07081286

Rice, F., Harold, G. T., Boivin, J., Van Den Bree1, M., Hay, D. F., \& Thapar, A. (2010). The links between prenatal stress and offspring development and psychopathology: Disentangling environmental and inherited influences. Psychological Medicine, 40, 335-345.

Shannon, K. E., Beauchaine, T. P., Brenner, S. L., Neuhaus, E., \& Kopp, L. G. (2007). Familial and temperamental predictors of resilience in children at risk for conduct disorder and depression. Developmental Psychopathology, 19(3), 701-727.

Shaw, D. S., Hyde, L. W., \& Brennan, L. M. (2012). Early predictors of boys' antisocial trajectories. Developmental Psychopathology, 24(3), 871-888.

Shaw, D. S., Lacourse, E., \& Nagin, D. S. (2005). Developmental trajectories of conduct problems and hyperactivity from ages 2 to 10. Journal of Child Psychology and Psychiatry, 46(9), 931-942.

Silk, J. S., Shaw, D. S., Skuban, E. M., Oland, R. A., \& Kovacs, M. (2006). 
Emotion regulation strategies in offspring of childhood-onset depressed mothers. Journal of Child Psychology and Psychiatry, 47(1), 69-78.

Sourander, A., Pihlakoski, L., Aromaa, M., Rautava, P., Helenius, H., \& Silanpää, M. (2006). Early predictors of parent- and self-reported perceived global psychological difficulties among adolescents. A prospective cohort study from age 3 to age 15. Social Psychiatry and Psychiatric Epidemiology, 41, 173-182.

Sterba, S. K., Prinstein, M. J., \& Cox, M. J. (2007). Trajectories of internalizing problems across childhood: heterogeneity, external validity, and gender differences. Development and Psychopathology, 19, 345-366.

Talati, A., Wickramaratne, P. J., Pilowsky, D. J., Alpert, J. E., Cerda, G., Garber, J., ... Weissman, M. M. (2007). Remission of maternal depression and child symptoms among single mothers: A STAR*D-child report. Social Psychiatry and Psychiatric Epidemiology, 42(12), 962-71. doi: 10.1007/ s00127-007-0262-4

Tompson, M. C., Pierre, C. B., Boger, K. D., McKowen, J. W., Chan, P. T., \& Freed, R. D. (2010). Maternal depression, maternal expressed emotion, and youth psychopathology. Journal of Abnormal Child Psychology, 38(1), 105-117. van der Molen, E., Hipwell, A. E., Vermeiren, R., \& Loeber, R. (2011). Maternal characteristics predicting young girls' disruptive behavior. Journal of Clinical Child \& Adolescent Psychology, 40(2), 179-190.

Vasa, R. A., Roberson-Nay, R., Klein, R. G., Mannuzza, S., Moulton, J. L., Guardino, M., \& Pine, M. D. (2007). Memory deficits in children with and at risk for anxiety disorders. Depression and Anxiety, 24, 85-94. doi: 10.1002/da.20193

Warner, V., Wickramaratne, P., \& Weissman, M. M. (2008). The role of fear and anxiety in the familial risk for major depression: a three-generation study. Psychological Medicine, 38, 1543-1556.

Weissman, M. M., Wickramaratne, P., Nomura, Y., Warner, V., Pilowsky, D., \& Verdeli, H. (2006). Offspring of depressed parents: 20 years later. American Journal of Psychiatry, 163, 1001-1008.

Wickramaratne, P., Gameroff, M. J., Pilowsky, D. J., Hughes, C. W., Garber, J., Malloy, ... Weissman, M. M. (2011). Children of depressed mothers 1 year after remission of maternal depression: Findings from the STAR*D-Child study. American Journal of Psychiatry, 168(6), 593-602. doi: 10.1176/appi. ajp.2010.10010032

Fernanda Aguiar Pizeta, doutoranda em Ciências - Saúde Mental pela Faculdade de Medicina de Ribeirão Preto, Universidade de São Paulo (FMRP-USP), é psicóloga do Tribunal de Justiça do Estado de São Paulo. E-mail: fepizeta@usp.br

Thaysa Brinck Fernandes Silva, mestranda em Ciências - Saúde Mental pela Faculdade de Medicina de Ribeirão Preto, Universidade de São Paulo (FMRP-USP). E-mail: thaysabrinck@hotmail.com Maria Isabela Brasil Cartafina, mestre em Ciências - Saúde Mental pela Faculdade de Medicina de Ribeirão Preto, Universidade de São Paulo (FMRP-USP). E-mail: isacartafina@gmail.com

Sonia Regina Loureiro, doutora em Psicologia Clínica pelo Instituto de Psicologia da Universidade de São Paulo (IPUSP), é professora doutora na Faculdade de Medicina de Ribeirão Preto, Universidade de São Paulo (FMRP-USP). E-mail: srlourei@fmrp.usp.br 